\title{
Producción, Circulación y Comunicación del conocimiento en Instituciones de Educación Superior: Políticas Editoriales Proyecto de Investigación \\ $\mathrm{N}^{\circ} 6.3$
}

Equipo de Investigación ${ }^{(1)}$

Ivana Mihal

por Facultad de Diseño y Comunicación, Universidad de Palermo y Laboratorio de Investigación en Ciencias Humanas de la UNSAM-CONICET (ARG)

Ana Elisa Ribeiro

por Centro Federal de Educacao Tecnológica de Minas Gerais-CEFET-MG (BRA)

Daniela Szpilbarg

por Instituto de Desarrollo Económico y Social -IDES- CONICET (ARG)

Resumen: El Proyecto de Investigación 6.3 propone recuperar tanto la trayectoria de editoras y actores del mundo editorial, como reflexionar sobre la condición de la autoría femenina, el sexismo en las prácticas editoriales, las desigualdades de género y las políticas del lenguaje en Argentina, Brasil y Uruguay, países en los que, si bien el devenir de la acción de las mujeres en el campo de la edición ha sido distinto, ha estado atravesado por condicionamientos, obstáculos, invisibilidades y desigualdades en torno a ellas. El campo de estudio en edición y cultura escrita reporta aún un área de vacancia del rol de las mujeres en el mundo del libro y particularmente su accionar como editoras. Este necesario reposicionamiento de la mirada en las ausencias que han plagado la construcción de la historia del libro y del mundo editorial contemporáneo es clave para componer un panorama de la edición de libros desde una perspectiva feminista, que recupere y visibilice la acción y el trabajo de ciertas mujeres que protagonizaron los devenires en los distintos países latinoamericanos.

Palabras clave: Campo editorial - América Latina - Trayectorias - Feminismo.

[Resúmenes en inglés y portugués en la página 183]

${ }^{(1)}$ Los CVs del Equipo de Investigación pueden consultarse en el Capítulo Directores de Líneas y Coordinadores de Proyectos de esta misma Edición. 


\section{Acerca del Proyecto 6.3}

\section{Producción, Circulación y Comunicación del conocimiento en Instituciones de Educación Superior Políticas Editoriales}

El Proyecto 6.3 Producción, Circulación y Comunicación del conocimiento en Instituciones de Educación Superior Políticas Editoriales, explora el universo editorial de la región, haciendo foco sobre el panorama de la mujer como autora y la mujer como editora. Surge como resultado de las discusiones, reflexiones y preocupaciones compartidas en torno al rol, la acción y la presencia de las mujeres y otras disidencias en el marco de los estudios de la lectura y la edición, entre el Programa "Mundo editorial, lectura y traducción desde los estudios de género(s) y feminismos" inscripto en el Centro de Estudios Interdisciplinarios en Educación, Cultura y Sociedad (CEIECS) que integra Laboratorio Interdisciplinario de Ciencias Humanas (LICH), unidad ejecutora del Consejo Nacional de Investigaciones Científicas y Técnicas (CONICET) de la Universidad Nacional de San Martín (UNSAM) en Argentina y el Grupo de Estudos Mulheres na Edição, del Centro Federal de Educação Tecnológica de Minas Gerais, Brasil.

Se acordó entre la Universidad de Palermo (Argentina), Centro Federal de Educacao Tecnológica de Minas Gerais-CEFET-MG (Brasil) y el Instituto de Desarrollo Económico y Social, IDES-CONICET (Argentina), avanzar en una investigación en la que participan investigadores, provenientes de distintas disciplinas, como comunicación, diseño, historia, antropología, sociología, ciencias de la educación, entre otras carreras afines, con la coordinación compartida de Ivana Mihal (UP-UNSAM-CONICET), Ana Elisa Ribeiro (CEFET-MG) y Daniela Szpilbarg (IDES-CONICET), con el fin de generar un espacio de reflexión crítica en torno a las desigualdades y dinámicas de poder presentes en el ejercicio femenino de la edición y la escritura, para restituir la importancia del rol de la mujer en la producción editorial, y de este modo impactar sobre la definición de los espacios de campo social de las nuevas generaciones de Diseñadore/as, Editore/as y Autore/as según una perspectiva de género.

Las reflexiones y los resultados obtenidos en el Proyecto 6.3 Producción, Circulación y Comunicación del conocimiento en Instituciones de Educación Superior: Políticas Editoriales, son continuación de los Proyectos 6.2 Edición universitaria y políticas editoriales como objeto de análisis, y el Proyecto 6.1 Lectura Digital: Incidencia de la práctica digital en los hábitos de lectura, en el marco de la misma Línea de Investigación y bajo la misma Directora Ivana Mihal.

Sus principales objetivos son:

- Estudiar los ámbitos concretos de abordaje del diseño, la comunicación, la edición y la autoría de libros, como su producción, circulación y políticas editoriales en la región, haciendo foco sobre la mujer como autora y la mujer como editora - Analizar la presencia de las mujeres en el mundo del libro y su reflexión epistemológica, en la construcción de una sociología histórica de las ausencias 
- Explorar los interrogantes acerca de las desigualdades, invisibilizaciones y dinámicas de poder en relación con la presencia femenina y otras disidencias en el campo de la edición y la autoría

- Estudiar, desde distintas perspectivas, la incidencia en el diálogo de libros, autores y lecturas con el diseño y la comunicación, en relación con la idea de violencia simbólica como parte de las estrategias desplegadas en contra de los espacios de acción de la mujer

- Ahondar en los consumos culturales de la sociedad contemporánea, mediante el análisis del sector editorial de libros y la idea del Estado en su deuda con los vulnerables

- Establecer bases para la construir la necesidad de revertir los equilibrios en la adquisición del capital simbólico de la mujer, en pos de su autonomía y adquisición de derechos

- Impactar en el Diseño Editorial, con la actualización de los conocimientos transitados en la carrera de Diseño Gráfico en relación con la perspectiva de género

\section{Acerca de la Línea 6}

Convergencia pedagógica digital: Miradas interdisciplinarias dirigida por Ivana Mihal se desarrolla de manera ininterrumpida desde 2015 en la Facultad de Diseño y Comunicación (UP, Argentina), e incluye hasta el momento tres proyectos finalizados el 6.1 Lectura Digital: Incidencia de la práctica digital en los hábitos de lectura coordinado por Ivana Mihal, el 6.2 Edición universitaria y políticas editoriales como objeto de análisis coordinado por Ivana Mihal (UP) y Daniela Szpilbarg (Instituto de Desarrollo Económico y Social, IDES-CONICET, Argentina), y el 6.3 Producción, Circulación y Comunicación del conocimiento en Instituciones de Educación Superior: Políticas Editoriales, coordinado por Ivana Mihal (Universidad de Palermo, y Laboratorio de Investigación en Ciencias Humanas de la UNSAM-CONICET, Argentina), Ana Elisa Ribeiro (Centro Federal de Educacao Tecnológica de Minas Gerais-CEFET-MG, Brasil) y Daniela Szpilbarg (Instituto de Desarrollo Económico y Social, IDES-CONICET, Argentina).

\section{Mapa de Áreas y Proyectos}

El Proyecto 6.3 se vincula con todas las carreras de grado y posgrado correspondientes a la Facultad de Diseño y Comunicación dado que impacta en la incorporación de la investigación en la lógica de la docencia universitaria, y específicamente de manera fundamental con la carrera de Diseño gráfico y Comunicación digital. Además los proyectos de la Línea de Investigación están vinculados también con los de la Línea de Investigación N9 Enseñar Disciplinas Proyectuales. 


\section{Productos y Resultados}

\section{a)- Publicaciones}

Cuaderno del Centro de Estudios de Diseño y Comunicación No107. (2020/2021) Editoras y autorías: las mujeres en el mundo editorial latinoamericano. Facultad de Diseño y Comunicación Año XXIII, Buenos Aires, Argentina. ISSN: 1668-0227.

Esta publicación documenta y comunica los resultados alcanzados en el Proyecto 6.3 Producción, Circulación y Comunicación del conocimiento en Instituciones de Educación Superior: Políticas Editoriales (finalizado), y a continuación se detallan los autores y artículos contenidos en ella:

Mihal Ivana, Ribeiro Ana Elisa, Szpilbarg Daniela (2020/2021) Introducción: "Editoras y autorías: las mujeres en el mundo editorial latinoamericano" (Pp. 11 a 16)

Mota de Almeida Lorrany, Melo Moreira Paula Renata (2020/2021) Literatura Juvenil de Mulheres Negras - Brasil, Século XXI (Pp. 17 a 34)

Páez Daniela (2020/2021) Las historietistas argentinas. Trayectorias, espacios y dinámicas de trabajo desde los ' 40 a la actualidad (Pp. 35 a 63)

Ribeiro Ana Elisa (2020/2021) Mulheres na edição: o caso de Tânia Diniz e o mural Mulheres Emergentes (Pp. 65 a 79)

Riveiro María Belén (2020/2021) Ada Korn editora: por una historia crítica del mundo editorial (Pp. 81 a 94)

Santana Gomes Letícia, Silva Giani David (2020/2021) Mulheres editoras independentes: Constanza Brunet (Argentina) Maria Mazarello (Brasil) e Paula Anacaona (França) e as projeções de si (Pp. 95 a 111)

Torres Torres Alejandra T (2020/2021) Mujeres editoras en el Uruguay contemporáneo: de Susana Soca (1906-1959) a Nancy Bacelo (1931-2007) (Pp.113 a 127) Gerbaudo Analía, Tosti Ivana (2020/2021) Beatriz Sarlo y el campo editorial en Argentina (Pp. 129 a 159)

Bombini Gustavo (2020/2021) Disidencia, resistencia y reposicionamiento: la actividad editorial entre dictadura y democracia. Mujeres editoras (Pp. 161 a 176)

A. Pereira Maria do Rosário (2020/2021) Narrativa brasileira de autoria feminina contemporânea: breves apontamentos (Pp. 177 a 192)

\section{b)- Congresos / Coloquios / Plenarios}

V Coloquio de Investigación y Desarrollo en Diseño Latino. Universidad de Palermo, 27 de julio de 2020. XI Congreso Latinoamericano de Enseñanza del Diseño. Semana Internacional del Diseño en Palermo.

En la comisión Edición Universitaria y Políticas Editoriales, se presentaron las reflexiones y conclusiones del proyecto 6.3 Producción, Circulación y Comunicación del conocimiento en Instituciones de Educación Superior: Políticas Editoriales (Finalizado) expusieron: Lorrany Mota de Almeida, Paula Renata Melo Moreira, Daniela Paéz, Ana Elisa Ribeiro, María Belén Rivero, Letícia Santana Gomes, Giani David Silva, Letícia Santana Gomes, 
Giani David Silva, Alejandra Torres Torres, Analia Gerbaudo, Ivana Tosti, Gustavo Bombini, Maria do Rosário Pereira, e Ivana Mihal.

A continuación se detallan las ponencias presentadas en esta Comisión coordinada por la Directora del proyecto Ivana Mihal, Ana Elisa Ribeiro y Daniela Szpilbarg:

Lorrany Mota de Almeida y Paula Renata Melo Moreira (Brasil)

Literatura juvenil de mujeres negras - Brasil, Siglo XXI

Daniela Paéz (Argentina)

Las historietistas argentinas. Trayectorias, espacios y dinámicas de trabajo desde los '40 a la actualidad

Ana Elisa Ribeiro (Brasil)

Mulheres na edição: o caso de Tânia Diniz e o mural Mulheres Emergentes

María Belén Rivero (Argentina)

Ada Korn editora: por una historia crítica del mundo editorial

Letícia Santana Gomes y Giani David Silva (Brasil)

Mulheres editoras independentes: o caso de três editoras e as projeções de si

Alejandra Torres Torres (Uruguay)

Trayectorias de editoras y actorxs del mundo editorial: mujeres editoras en el Uruguay contemporáneo: de Susana Soca (1906-1959) a Nancy Bacelo (1931-2007)

Analia Gerbaudo e Ivana Tosti (Argentina)

Beatriz Sarlo y el campo editorial en Argentina

Gustavo Bombini (Argentina)

Disidencia, resistencia y reposicionamiento: la actividad editorial entre dictadura y democracia: Mujeres editoras

Maria do Rosário Pereira (Brasil)

Narrativa brasileña de autoría femenina contemporánea: notas breves

\title{
c)- Formación de Posgrado e Impacto curricular
}

La Directora Ivana Mihal es Docente de Posgrado en Diseño de la Facultad de Diseño y Comunicación en Maestría en Gestión del Diseño y Doctorado en Diseño y al igual que Daniela Spilzbarg (IDES-CONICET, ARG) y Ana Elisa Ribeiro (CEFET-MG, BRA) en sus respectivas Instituciones, incorporan los contenidos de su investigación a sus asignaturas en posgrado.

\begin{abstract}
Research Project 6.3 proposes to recover both the trajectory of editors and actors in the publishing world, as well as to reflect on the condition of female authorship, sexism in editorial practices, gender inequalities and language policies in Argentina, Brazil and Uruguay, countries in which, although the evolution of the action of women in the field of publishing has been different, it has been crossed by conditions, obstacles, invisibilities and inequalities around them. The field of study in publishing and written culture still reports an area of vacancy in the role of women in the world of books and particularly
\end{abstract}


their actions as editors. This necessary repositioning of the gaze in the absences that have plagued the construction of the history of the book and the contemporary publishing world is key to compose a panorama of book publishing from a feminist perspective, which recovers and makes visible the action and the work of certain women who starred in the events in the different Latin American countries.

Keywords: Editorial field - Latin America - Trajectories - Feminism

Resumo: O Projeto de Pesquisa 6.3 se propõe a resgatar a trajetória de editores e atores do mundo editorial, bem como refletir sobre a condição da autoria feminina, o sexismo nas práticas editoriais, as desigualdades de gênero e as políticas linguísticas na Argentina, Brasil e Uruguay, países nos quais, embora a evolução da ação das mulheres no campo da edição tenha sido diferente, ela foi atravessada por condições, obstáculos, invisibilidades e desigualdades ao seu redor. O campo de estudos em cultura editorial e escrita ainda relata uma vaga área do papel da mulher no mundo do livro e, principalmente, sua atuação como editora. Este necessário reposicionamento do olhar nas ausências que têm atormentado a construção da história do livro e do mundo editorial contemporâneo é fundamental para compor um panorama da edição de livros numa perspectiva feminista, que recupere e torne visível a ação e a trabalho de algumas mulheres que protagonizaram eventos em diversos países da América Latina.

Palavras chave: Campo editorial - América Latina - Trajetórias - Feminismo.

[Las traducciones de los resúmenes fueron realizadas a través de traductor automático] 